\title{
SOLUTION OF SOME PROBLEMS OF SINGLE-SCALE WAVELET TRANSFORM PROCESSOR USING A MAGNETOSTATIC SURFACE WAVE DEVICE
}

\author{
Wenke Lu'), Lun Kuang ${ }^{4)}$, Xiaozhou Lü ${ }^{2)}$,Changchun Zhu'), Ting Zhang ${ }^{4)}$, \\ Jingduan Zhang ${ }^{1)}$ \\ 1) Donghua University, School of Information science and Technology, Shanghai 201620, China, \\ (\luwenkelu@163.com, +8602167792312, zhangjingduan2@163.com) \\ 2) Xidian University, School of Mechano-Electronic Engineering, Xi'an 710071, China, (lxz@uw.edu) \\ 3) Xi'an Jiaotong University, School of Electronics and Information Engineering, Xi'an 710049, China, (chzh@mail.xjtu.edu.cn) \\ 4) Beijing of Radio Measurement, Beijing 100854,China, (kuanglun@163.com,_zhang_x@126.com)
}

\begin{abstract}
In this paper, we investigate the implementation schemes of a single-scale wavelet transform processor using magnetostatic surface wave (MSSW) devices. There are three implementation schemes: the interdigital transducer, the meander line transducer and the grating transducer. Because the interdigital transducer has excellent properties, namely, good frequency characteristic and low insertion loss, we use the interdigital transducer as the implementation scheme of a single-scale wavelet transform processor using MSSW device.

In the paper, we also present the solutions to the three key problems: the direct coupling between the input transducer and the output transducer, the insertion loss, and the loss characteristics of the gyromagnetic film having an influence on the wavelet transform processor. There are two methods of reducing the direct coupling between the input transducer and the output transducer: increasing the distance between the input transducer and the output transducer, and placing a metal "wall" between the input transducer and the output transducer. There also are two methods of reducing the insertion loss of a single-scale wavelet transform processor using a MSSW device for scale: the appropriate thickness of the yttrium iron garnet (YIG) film and the uniform magnetic field.The smaller the ferromagnetic resonance linewidth of the gyromagnetic film, the smaller the magnetostatic wave propagation loss.
\end{abstract}

Keywords: wavelet transform processor, magnetostatic surface wave (MSSW), interdigital transducer, direct coupling, insertion loss.

(C) 2012 Polish Academy of Sciences. All rights reserved

\section{Introduction}

Wavelet transform finds its application in many disciplines and fields such as in pronunciation, pictures, communication, radar, water-sound, earthquakes, biomedicine, mechanical vibration, chemical industry and torrent analysis. It has pushed the information industry to a new era. However, its algorithms are very complicated and difficult to implement in engineering application fields. In order to solve this problem, many methods such as very large scale integration (VLSI) [1-2], optical devices [4-5] and SAW devices [6-8] have been employed in implementing a wavelet transform processor.

In the method of implementation of wavelet transform with SAW devices, [6] and [7] found that, if the electrode-overlap envelope of the input interdigital transducer (IDT) for SAW device is designed according to the envelope of wavelet function, the input IDT of the SAW device can implement the convolution of $f(t)$ and wavelet function, i.e. wavelet transform. Also [6] and [8] proposed the implementation of wavelet inverse-transform with SAW devices. 
The wavelet transform processor and wavelet inverse-transform processor that use SAW devices can benefit from the excellent properties of the SAW devices: passive, small size, low cost, excellent temperature stability, high reliability and reproducibility, which overcome the complicated algorithms and high power for VLSI, and big size and low reproducibility for optical devices, but in microwave band, the propagation losses of the wavelet transform processor and wavelet inverse-transform processor using SAW devices are very high, and their transducer fabrication is also difficult [9-12].

Magnetostatic Surface Wave Devices (MSSW) were developed in the 80's . These devices were applied as delay lines and filters in radar technology [13-15]. In this research, MSSW devices are used to implement the wavelet transform processor in the microwave band, and the wavelet transform processor using MSSW devices can benefit from the excellent properties of the MSSW devices which have low propagation loss and simple transducers (as shown in Table 1). In Table 1, the propagation loss of a MSSW device is much lower than that of a SAW device, and the conducing strip width of the MSSW device is much wider than the one of a SAW device (i.e. the transducers of the wavelet transform processor using MSSW devices are easily fabricated). When the envelope of the conducting strips of the input transducer for a MSSW device is designed according to the wavelet-function envelope, the impulse-response function of the input transducer for the MSSW device is equal to a wavelet function, so a single-scale wavelet transform processor using a MSSW device can be fabricated.

In this paper, we investigate the implementation schemes of single-scale wavelet transform processors using magnetostatic surface wave (MSSW) devices, and we also present the solutions to the three key problems: the direct coupling between the input transducer and the output transducer, the insertion loss, and the loss characteristics of the gyromagnetic film having an influence on the wavelet transform processor.

Table 1. Comparison of parameters of SAW devices and MSSW devices.

\begin{tabular}{|l|l|l|}
\hline & SAW device & MSSW device \\
\hline frequency & $10 \mathrm{MHz} 3 \mathrm{GHz}$ & $0.5 \mathrm{GHz} \sim 40 \mathrm{GHz}$ \\
\hline Substrate & $128^{0} \mathrm{y} / \mathrm{x}-\mathrm{LiNbO}$ substrate & yttrium-iron-garnet (YIG) film \\
\hline Propagation loss & $100 \mathrm{~dB} / \mu \mathrm{s}(10 \mathrm{GHz})$ & $12 \mathrm{~dB} / \mu \mathrm{s}(10 \mathrm{GHz})$ \\
\hline Conducing strip width & $0.099 \mu \mathrm{m}(5 \mathrm{GHz})$ & $40 \mu \mathrm{m}(5 \mathrm{GHz})$ \\
\hline Spacing between the conducing strips & $0.099 \mu \mathrm{m}$ & $310 \mu \mathrm{m}$ \\
\hline
\end{tabular}

This paper is organized as follows. After this introductory section, in Section 2 we propose the implementation schemes of a single-scale wavelet transform processor using a MSSW device. In Section 3, we give the solutions to three key problems of the wavelet transform processor. Conclusions are drawn in Section 4.

\section{Implementation schemes of a single-scale wavelet transform processor using a MSSW device}

The wavelet function is [6-14]:

$$
\psi_{s}(t)=\frac{1}{\sqrt{s}} \psi\left(\frac{t}{s}\right)
$$

where $s$ denotes the scale of the wavelet function.

The wavelet transform of signal $f(t)$ is [6-14]. 


$$
\begin{aligned}
W T_{s}(\tau) & =f(t) * \psi_{s}(t)=\int_{R} f(t) \frac{1}{\sqrt{s}} \psi\left(\frac{\tau-t}{s}\right) d t \\
& =\frac{1}{\sqrt{s}} \int_{R} f(t) \psi\left(\frac{\tau-t}{s}\right) d t .
\end{aligned}
$$

In (1), when $\psi_{s}(t)$ is a Morlet wavelet function, (1) is converted into

$$
\psi_{s}(t)=\frac{1}{\sqrt{s}} e^{-\frac{1}{2}\left(\frac{t}{s}\right)^{2}} e^{j 2 \pi \frac{f_{0}}{s} t},
$$

where $\frac{1}{\sqrt{s}} e^{-\frac{1}{2}\left(\frac{t}{s}\right)^{2}}$ is the envelope of the Morlet wavelet function, $\frac{f_{0}}{s}$ is the center frequency.

When $s=2^{k}$, the wavelet function shown in (3) is converted into the Morlet dyadic wavelet function [6-12].

The microwave communication equipment of our design needs a single-scale wavelet transform processor with the center frequency $3.830 \mathrm{GHz}$, bandwidth $48.000 \mathrm{MHz}$ and insertion loss $-17 \mathrm{~dB}$ as a band-pass filter. Through analysis and research we found that , as long as the input transducer of MSSW device is designed according to a scale wavelet function shown in the formula (4), a single-scale wavelet transform processor using a MSSW device can be implemented. We know from [6-12] that a single-scale wavelet transform processor using the Morlet wavelet function can be used as a band-pass filter.

$$
\begin{gathered}
\psi_{s}(t)=\frac{1}{\sqrt{s}} e^{-\frac{1}{2}\left(\frac{t}{s}\right)^{2}} e^{j 2 \pi \frac{f_{0}}{s} t} \\
=\psi_{1.492^{k}}(t)=\frac{1}{\sqrt{1.492^{k}}} e^{-\frac{1}{2}\left(\frac{t}{1.492^{k}}\right)^{2}} e^{-j 2 \pi \frac{f_{0}}{1.492^{k}} t},
\end{gathered}
$$

where the scale $s=1.492^{k}, \frac{f_{0}}{1.492^{k}}$ is the center frequency, $k$ is an integer from $-\infty$ to $+\infty$. ${ }^{13}$ is:

When $k$ is -13 , the scale $s$ is equal to $1.492^{-13}$, so the wavelet function for the scale $1.492^{-}$

$$
\begin{aligned}
& \psi_{1.492^{-13}}(t)=13.474 e^{-16480083 t^{2}} e^{-j 2 \pi 1.492^{13} f_{0} t} \\
& =13.474 e^{-16480083 t^{2}} e^{-j 2 \pi f_{-13} t},
\end{aligned}
$$

where $f_{-13}$ is the center frequency for the scale $1.492^{-13}, f_{-13}=1.492^{13} f_{0}=3.830 G H_{Z}$.

The wavelet transform of signal $f(t)$ for the scale $1.492^{-13}$ is:

$$
\begin{gathered}
W T_{1.492^{-13}}=f(t) * \psi_{1.492^{-13}}(t) \\
=\int_{R} f(t) \psi_{1.492^{-13}}(\tau-t) d t \\
=\int_{R} f(t) 13.474 e^{-16480083(\tau-t)^{2}} e^{j 2 \pi f_{-13}(\tau-t)} d t .
\end{gathered}
$$

We take the single-scale wavelet transform processor for scale $1.492^{-13}$ as an example to illustrate the design method of the single-scale wavelet transform processor (as shown in Fig. $1)$. 


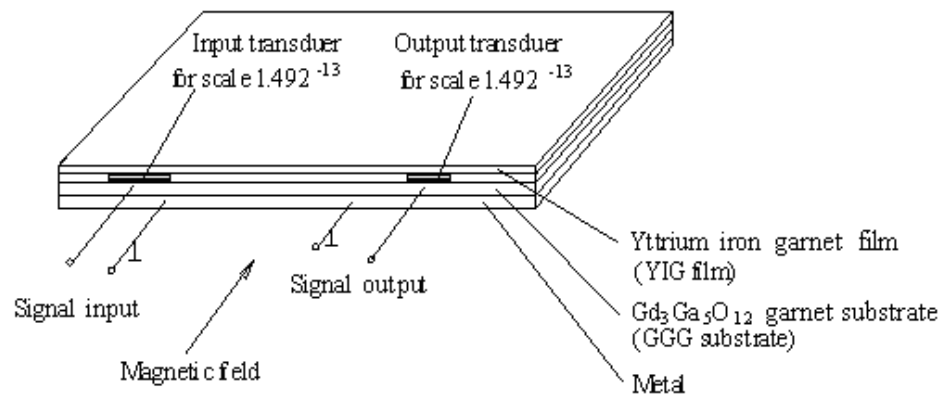

Fig. 1. Schematic diagram of a single-scale wavelet transform processor using a MSSW device.

There are three implementation schemes of the input transducer for scale $1.492^{-13}$ : the interdigital input transducer, the meander line input-transducer, and the grating inputtransducer. We will discuss the three implementation schemes of the input transducer for scale $1.492^{-13}$ in detail, as shown below.

Fig. 2 is the implementation scheme of the interdigital input transducer. The interdigital input transducer has excellent properties, namely, good frequency characteristics and low insertion loss. Fig. 3 and Fig. 4 are respectively the meander line input-transducer and the grating input-transducer. When the properties of the meander line input-transducer and the grating input-transducer are compared with those of the interdigital input transducer, their properties are relatively poor.

Through the above analysis and research, the implementation scheme of the interdigital input transducer is applied to fabricate the single-scale wavelet transform processor using a MSSW device in the paper.

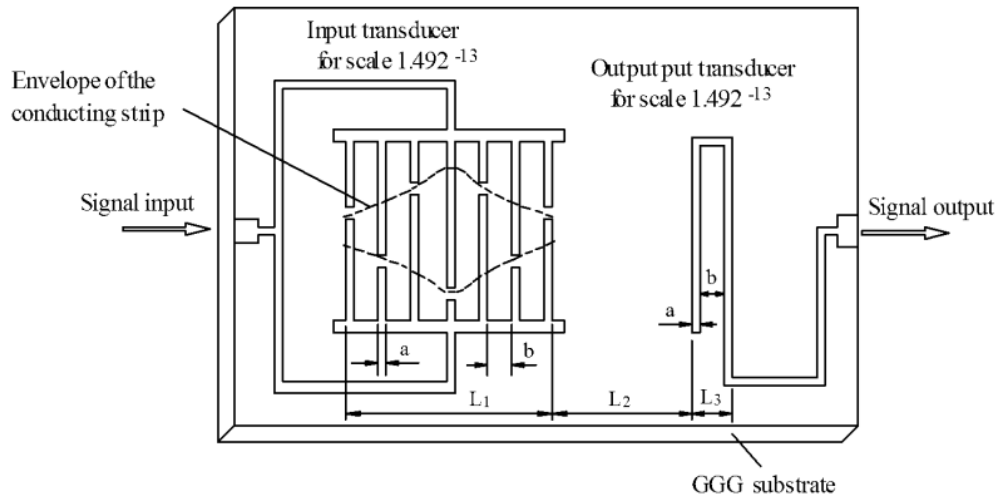

Fig. 2. Implementation scheme of the interdigital input transducer.

The transducer substrate of scale $1.492^{-13}$ shown in Fig. 2 is fabricated on a GGG substrate. The fabricated transducer substrate of scale $1.492^{-13}$ is shown pasted in Fig.1.

In Fig. 2, when the conducting-strip envelope of the input transducer for scale $1.492^{-13}$ is designed according to the envelope (i.e. $13.474 e^{-16480083 t^{2}}$ ) of the wavelet function $\psi_{1.492^{-13}}(t)$, the impulse-response function $h_{1.492^{-13}}(t)$ of the input transducer for scale $1.492^{-13}$ is equal to the wavelet function $\psi_{1.492^{-13}}(t)$ of scale $1.492^{-13}$, so the input transducer of scale $1.492^{-13}$ can implement the wavelet transform of scale $1.492^{-13}$ shown in formulas (6), i.e. the convolution of $f(t)$ and $\psi_{1.492^{-13}}(t)$. The output transducer of scale $1.492^{-13}$ only converts the MSSW signal into an electrical signal.

The design parameters of the input transducer and output transducer of scale $1.492^{-13}$ shown in Fig. 2 are presented in Table 2. 


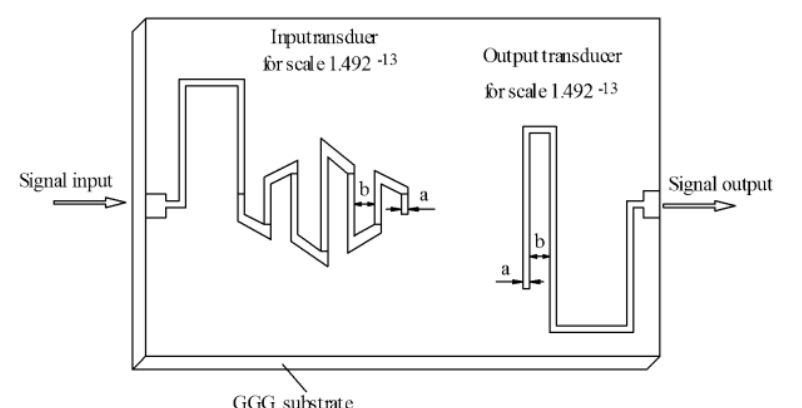

Fig. 3. Implementation scheme of the meander line input transducer.

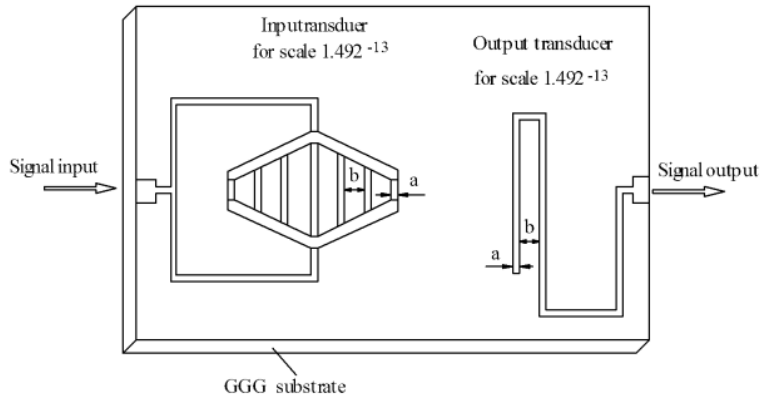

Fig. 4. Implementation scheme of the grating input-transducer.

Table 2. Design parameters of input transducer and output transducer of scale $1.492^{-13}$.

\begin{tabular}{|c|c|c|c|c|c|c|c|c|c|c|}
\hline \multirow[b]{2}{*}{ Scale } & \multirow[b]{2}{*}{ substrate } & \multirow{2}{*}{$\begin{array}{c}\text { Propagating } \\
\text { velocity of } \\
\text { MSSW } \\
(\mathrm{km} / \mathrm{s})\end{array}$} & \multirow{2}{*}{$\begin{array}{c}\text { Centre } \\
\text { frequency } \\
\left(\mathrm{GH}_{\mathrm{Z}}\right)\end{array}$} & \multirow{2}{*}{$\begin{array}{c}-3 \mathrm{~dB} \\
\text { bandwidth } \\
\left(\mathrm{MH}_{\mathrm{Z}}\right)\end{array}$} & \multirow{2}{*}{$\begin{array}{l}\text { inserti } \\
\text { on } \\
\text { loss } \\
(\mathrm{dB})\end{array}$} & \multirow{2}{*}{$\begin{array}{l}\text { Width of } \\
\text { conducting } \\
\text { strips } \\
\text { (the } \\
\text { conducting } \\
\text { strips) }\end{array}$} & \multirow{2}{*}{$\begin{array}{c}\text { Spacing } \\
\text { between the } \\
\text { conducting } \\
\text { strips }(\mu \mathrm{m})\end{array}$} & \multicolumn{2}{|c|}{$\begin{array}{l}\text { Number of the } \\
\text { conducting strips }\end{array}$} & \multirow{2}{*}{$\begin{array}{c}\text { Envelope of } \\
\text { conducting strips }\end{array}$} \\
\hline & & & & & & & & $\begin{array}{c}\text { Input } \\
\text { transducer }\end{array}$ & $\begin{array}{l}\text { Output } \\
\text { transducer }\end{array}$ & \\
\hline 1. $492^{-13}$ & $\begin{array}{c}\text { YIG film } \\
\text { (thickness } \\
\text { of YIG is } \\
235 \mu \mathrm{m} \text { ) }\end{array}$ & 142 & 3.830 & 48.030 & -17 & 40 & 310 & 11 & 2 & $13.474 e^{-16480.0}$ \\
\hline
\end{tabular}

\section{Solutions to three problems of the wavelet transform processor}

\subsection{Solution to direct coupling between the input transducer and the output transducer}

The direct coupling between the input transducer and the output transducer can produce pass-band ripples, cause amplitude and phase distortions, and also increase the insertion loss. There are two methods of reducing the direct coupling between the input transducer and the output transducer: increasing the distance between the input transducer and the output transducer, and placing a metal "wall" between the input transducer and the output transducer. When the width of the input transducer is smaller than the distance between the input transducer and the output transducer, the problem of direct coupling between the input transducer and the output transducer can be solved. The width $\mathrm{L}_{1}$ of input transducer is $3540 \mu \mathrm{m}$, the distance $\mathrm{L}_{2}$ between the input transducer and the output transducer is $8000 \mu \mathrm{m}$, as shown in Fig. 2.

When a metal "wall" is placed between the input transducer and the output transducer, the direct coupling between the input transducer and the output transducer can be reduced and the resonant structure of the cavity also can be destroyed.

\subsection{Solutions to the insertion loss}

There are two methods of reducing the insertion loss of the single-scale wavelet transform processor using MSSW device of scale $1.492^{-13}$ : appropriate thickness of the YIG film, and a uniform magnetic field.

\subsubsection{Using an appropriate thickness of the YIG film to compensate the insertion loss}

We know that the greater the YIG film thickness of MSSW device, the smaller the insertion loss, on the other hand, the smaller the thickness of YIG film, the narrower the bandwidth of the MSSW device. Because the bandwidth of the single-scale wavelet transform 
processor using a MSSW device of scale $1.492^{-13}$ is $1.25 \%$, its bandwidth is smaller. Therefore, we must take both above requirements into account. In the paper, the YIG film thickness is $23.5 \mu m$, as shown in Table 2 .

\subsubsection{Using a uniform magnetic field to compensate the insertion loss}

In order to reduce the insertion loss caused by the anisotropy of MSSW energy propagation, we must apply a uniform magnetic field to the single-scale wavelet transform processor using a MSSW device of scale $1.492^{-13}$. When the length $L$ of the permanent magnet is much larger than the sum of the width $L_{1}$ of the input transducer, the width $L_{3}$ of the output transducer, and the distance $L_{2}$ between the input transducer and the output transducer (i.e. $L>L_{1}+L_{2}+L_{3}$, as shown in Fig. 2 ), the magnetic field produced by the permanent magnet is uniform.

\subsection{Loss characteristics of the gyromagnetic film having an influence on the wavelet transform processor}

The magnetostatic wave propagation loss is:

$$
I L(d B)=0.96 q \Delta H \tau,
$$

where $1 \leq q \leq\left[1+\left(\gamma M_{0} / 2 f_{0}\right)^{2}\right], M_{0}$ is the saturation magnetization of the gyromagnetic film (i.e. the YIG film), $\gamma$ is the gyromagnetic ratio constant, $f_{0}$ is the microwave frequency, $\Delta H$ is the ferromagnetic resonance linewidth of the gyromagnetic film , $\tau$ is the group delay from the input transducer to the output transducer.

We know from the formula (7) that $I L(d B)$ is directly proportional to $\Delta H$, i.e. the smaller $\Delta H$, the smaller $I L(d B)$. The insertion loss of MSSW strictly depends on the linewidth of the yttrium iron garnet (YIG). The current technology guaranty of this parameter is at the level of about $80-100 \mathrm{~A} / \mathrm{m}$. In this work, $\Delta H$ used is less than $180 \mathrm{~A} / \mathrm{m}$. When $q=1.3, \tau=0.05 \mu \mathrm{s}$, and $\Delta H=180 \mathrm{~A} / \mathrm{m}$, we get from the formula (7) that $I L(d B)=11.23 d B$ which meets the requirements of design and manufacturing.

\section{Experiments}

A single-scale wavelet-transform processor using a MSSW device of scale $1.492^{-13}$ was fabricated (as shown in Fig. 5). It was measured with the E838B network analyzer and its experimental parameters are shown in Table 3.

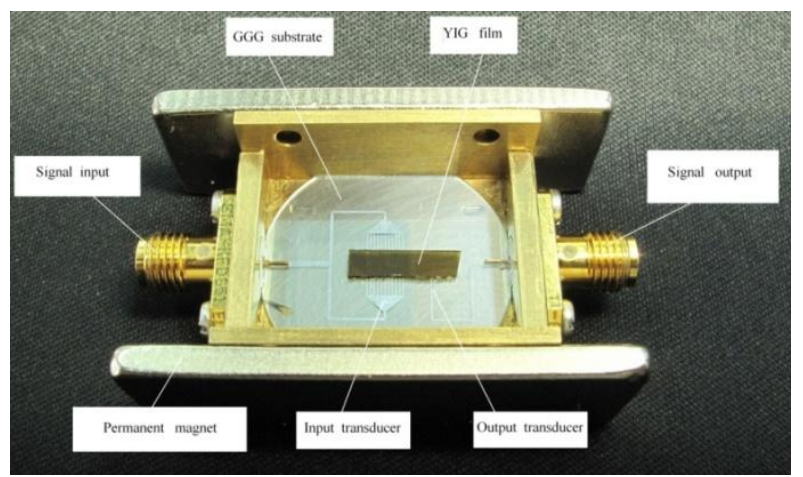

Fig. 5. Single-scale wavelet transform processor using a MSSW device of scale $1.492^{-13}$. 
Table 3. Comparison of experimental and theoretical parameters of the single-scale wavelet-transform processor using a MSSW device of scale $1.492^{-13}$.

\begin{tabular}{|l|l|l|l|l|l|l|l|l|l|l|}
\hline & \multirow{2}{*}{ Scale } & \multirow{2}{*}{$\begin{array}{l}\text { Experimental } \\
\text { insertion loss } \\
(\mathrm{dB})\end{array}$} & $\begin{array}{l}\text { Experimental } \\
\text { centre } \\
\text { frequency } \\
(\mathrm{GHz})\end{array}$ & \multicolumn{3}{|l|}{ Theoretical bandwidth $\left(\mathrm{MH}_{\mathrm{Z}}\right)$} & \multicolumn{3}{|l|}{ Experimental bandwidth $\left(\mathrm{MH}_{\mathrm{Z}}\right)$} \\
\cline { 5 - 11 } & & & $-3 \mathrm{~dB}$ & $-6 \mathrm{~dB}$ & $-9 \mathrm{~dB}$ & $-12 \mathrm{~dB}$ & $-3 \mathrm{~dB}$ & $-6 \mathrm{~dB}$ & $-9 \mathrm{~dB}$ & $-12 \mathrm{~dB}$ \\
\hline $1.492^{-13}$ & -15.180 & 3.843 & 48.030 & 67.924 & 83.190 & $\begin{array}{l}96 . \\
0599\end{array}$ & 48.042 & 66.357 & 77.785 & 104.361 \\
\hline
\end{tabular}

The theoretical bandwidths shown in Table 3 are the magnitudes obtained from (8), i.e.

$$
\psi_{1.492^{-13}}(\omega)=227.538 e^{-\frac{1}{65920332}\left(\omega-\omega_{-13}\right)^{2}},
$$

where $\omega_{-13}$ is the center frequency.

We know from Fig. 5 and Table 3 that the interdigital transducer of the MSSW device can implement a single-scale wavelet transform processor.

\section{Conclusion}

Theory and the experimental results confirm that the interdigital transducer of a MSSW device can implement a single-scale wavelet transform processor. In the paper we also present the solutions to the three key problems: the direct coupling between the input transducer and the output transducer, the insertion loss, and the loss characteristics of the gyromagnetic film having an influence on the wavelet transform processor.

There are two methods of reducing the direct coupling between the input transducer and the output transducer: increasing the distance between the input transducer and the output transducer, and placing a metal "wall" between the input transducer and the output transducer. When the width of the input transducer is smaller than the distance between the input transducer and the output transducer, the problem of the direct coupling between the input transducer and the output transducer can be solved. When a metal "wall" is placed between the input transducer and the output transducer, the direct coupling between the input transducer and the output transducer also can be reduced.

There are the two methods of reducing the insertion loss of a single-scale wavelet transform processor using a MSSW device: the appropriate thickness of the YIG film and a uniform magnetic field. We know that the greater the YIG film thickness of MSSW device, the smaller the insertion loss, on the other hand, the smaller the thickness of YIG film, the smaller the bandwidth of the MSSW device. Therefore, we must take account both above requirements. In the paper, the YIG film thickness is $23.5 \mu \mathrm{m}$. In order to reduce the insertion loss caused by the anisotropy of MSSW energy propagation, we must apply a uniform magnetic field to the single-scale wavelet transform processor using a MSSW device. When the length of the permanent magnet is much larger than the sum of the width of the input transducer, the width of the output transducer, and the distance between the input transducer and the output transducer, the magnetic field produced by the permanent magnet is uniform.

The smaller the ferromagnetic resonance linewidth of the gyromagnetic film, the smaller the magnetostatic wave propagation loss. 
W. Lu, L. Kuang, X. Lü, C. Zhu, T. Zhang, J. Zhang: SOLUTION OF SOME PROBLEMS OF SINGLE-SCALE WAVELET ...

\section{Acknowledgments}

This work was supported by the National Natural Science Foundation of China (Grant Nos.: 60976058, 61274078) and Innovation Program of Shanghai Municipal Education Commission (Grant No. 13ZZ049).

\section{References}

[1] Zhang, C., Wang, C., Ahmad, M.O. (2012). A Pipeline VLSI Architecture for Fast Computation of the 2-D Discrete Wavelet Transform. IEEE Transactions on Circuits and Systems I, 99(1), 1-11.

[2] Tian, X., Wu, L., Tan, Y.H., Tian, J.W. (2011). Efficient Multi-Input/Multi-Output VLSI Architecture for Two-Dimensional Lifting-Based Discrete Wavelet Transform. IEEE Transactions on Computers, 60(8), 1207-1211.

[3] Roberge, D., Sheng, Y. (1994) Optical wavelet matched filter. Applied Optics, 33(23), 5287-5293.

[4] Chen, X., Zhang, X., Chen, K., Li, Q. (1997). Optical wavelet-matched filtering with bacteriorhodopsin films. Applied Optics, 36(32), 8413-8416.

[5] Lu, W., Zhu, C., Liu, J., Liu, Q. (2003). Implementing wavelet transform with SAW elements. Science China, 46(6), 627-638.

[6] Lu, W., Zhu, C., Liu, Q., Liu, J. (2005). Wavelet transform element of SAW type. Chinese Science Bulletin, 50(6), 598-602.

[7] Lu, W., Zhu, C. (2011). A novel compensation method of insertion losses for wavelet inverse-transform processors using surface acoustic wave devices. Review of Scientific Instruments, 82(11), 115003.1115003.7.

[8] Lu, W., Zhu, C. (2010). Solving three key problems of wavelet transform processor using surface acoustic wave devices. IEEE Trans. Industrial Electronics, 57(11), 3801-3806.

[9] Wen, C., Zhu, C., Ju, Y., Qiu, Y., Lu, W., Hu, X. (2008). Dual track architecture and time synchronous scheme for wavelet reconstruction processor using SAW device based on MSC. Sens. Actuators A, 147(5), 222-228.

[10] Wen, C., Zhu, C., Ju, Y., Qiu, Y. Lu W., Hu, X. (2009). Optimal frequency band design scheme of dyadic wavelet processor array using surface acoustic wave devices. IEEE Trans. Industrial Electronics, 56(4), 949-955.

[11] Lu, W., Zhu, C. (2011). Study of small size wavelet transform processor and wavelet inverse-transform processor using SAW devices. Measurement, 44(5), 994-999.

[12] Sethares, J.C. (1982). Magnetostatic wave devices and applications. Journal of Applied Physics, 53(3), 2646-2651.

[13] Castera, J.P. (1984). State of the art in design and technology of MSW devices. Journal of Applied Physics, 55(6), 2506-2511.

[14] Ishak, W.S. (1988). Magnetostatic wave technology. In Proc. of the IEEE, 76(2), 171-187. 